\title{
Anatomical Causes of Female Obstructive Voiding: Urethral Stricture and Anti-Incontinence Procedures
}

\begin{abstract}
Obstructive voiding affects up to $29 \%$ of the female population. The iatrogenic causes of female uninary obstruction are preventable to an extent and deserve special consideration. These include urethra stric ture and obstruction following surg ery forstressurina ry inc ontinence. An understanding of the etiology and diagnosis of these two forms of obstruction is necessary for successful identification and management of these conditions. The most commonly used approaches to diagnosis include urethroscopy, voiding cystourethrography and flurourodynamics. Treatment methods proven successful for urethra stricture include dilatation, urethroplasty, flaps, and grafting. Common treatment approaches for obstruction following surgical correction of SUl include catheterization, urethrolysis, and sling incision. This review will further examine the etiology, diagnosis, and treatment of urethra stric ture and obstruction after surgery with the intent of contributing to a consensus on diagnostic approach and management of these conditions.
\end{abstract}

\section{Introduction}

Bladder outlet obstruction is not a common diagnosis among female patients. However, as the incidence of bladder manipulation increases in procedures used to treat incontinence, there is a corresponding increase in patients presenting with complications. There is still debate as to the true incidence of bladder obstruction, but estimates range from 2.7 to $29 \%$ [1]. Because we lack specific diagnostic criteria for women, there may be missed cases of obstruction, which can lead to improper treatment for these patients.

There are many different causes of obstruction in females. Awareness of possible iatrogenic sources is imperative in order to take appropriate steps in avoiding the complications of urethral manipulation. Two anatomical causes of obstruction that can occur iatrogenically include urethral stricture and obstruction following surgical repair for stress urinary incontinence.

Female urethral stricture is a rare disorder. However, total annual expenditure for this diagnosis was estimated to be $\$ 69$ million in 2000 [2]. Santucci et al. attribute this high cost to inadequate diagnosis or misdiagnosis of urethral stricture. Misdiagnosis then leads to inappropriate treatment [2]. Therefore, there is demand for standardization in the approach to patients with urethral stricture in order to decrease cost and lead to better patient outcomes. Review of literature suggests that female urethral stricture accounts for 4-13\% of cases of bladder outlet obstruction in women [3]. Symptoms associated with stricture include urinary frequency, urgency, dysuria, hesitancy, dribbling, incontinence, and recurrent urinary tract infections [4]. Blaivis et al. suggest that irritative symptoms, such as frequency and urgency, are more commonly manifested in the female presenting with urethral strictures [5]. Many institutions

\section{Journal of} Andrology \&
Gynaecology

\section{Katherine Cockerill, Katie Canalicio and Rowena} DeSouza*

Department of Urology, University of Texas at Houston Medical School, USA

\section{*Address for Correspondence}

Rowena DeSouza MD, Assistant Professor, Department of Urology, University of Texas at Houston Medical School, USA; E-mail: rowenadesouza@hotmail.com

\section{Submission: 27 May 2014}

Accepted: 18 June 2014

Published: 20 June 2014

Reviewed \& Approved by: Dr. Anastasios Athanasopoulos, Associate Professor of Urology and Head of Urodynamic Urology Unit at the Medical School in the University of Patras, Greece

have explored methods in addressing the diagnosis and treatment of female urethral stricture with varied results.

Stress urinary incontinence is a common condition affecting women, especially as the population ages. Common surgical approaches to this diagnosis include transobturator tape and retropubic sling procedures. However, surgical treatment is not without complications, which can range in presentation from transient to permanent urethral obstruction [6,7]. Nitti reports that the incidence of obstruction after incontinence surgery can range from 2.5 to $24 \%$ [1]. Obstruction after surgery may cause symptoms of hesitancy, decreased urinary flow, urgency, and frequency. In addition, other irritative symptoms such as pain, dysuria, and nocturia can occur. Petri and Ashok observed that obstructive symptoms more commonly occur with retropubic tapes compared to the transobturator tapes [8]. A high index of suspicion for obstruction is necessary for post-operative patients presenting with these lower urinary tract symptoms. There is still debate regarding diagnosis of post-surgical obstruction, and recent studies have also focused on proper management of obstruction in the post-surgical patient.

This paper will examine the etiology, diagnosis, and management of obstruction following anti-incontinence surgery and urethral stricture.

\section{Etiologies}

Both urethral stricture and obstruction following antiincontinence surgery are anatomical causes of bladder outlet obstruction in women [4]. Review of the anatomy of the female urethra affords insight into the mechanism of obstruction as well as approaches to repair of obstructive disease. The urethral wall is composed of a mucous membrane and two muscular layers, an inner longitudinal and outer circular layer. The muscular layer is continuous with the detrusor muscle of the bladder, and the mucous coat is continuous with that of the bladder trigone superiorly and vulva inferiorly. The undilated urethra measures $4 \mathrm{~cm}$ long and has a diameter of about $6 \mathrm{~mm}$. Anatomical obstruction of the urethra can occur from extrinsic, luminal or urethral origins. Female stricture 
Citation: Cockerill K, Canalicio K, DeSouza R. Anatomical Causes of Female Obstructive Voiding: Urethral Stricture and Anti-Incontinence Procedures. J Androl Gynaecol. 2014;2(1): 5.

and post-operative obstruction fall under urethral origins of bladder obstruction.

Urethral stricture can result from idiopathic, iatrogenic, traumatic, or inflammatory causes (Table 1) [3]. There is not sufficient statistical data regarding the prevalence of each etiology. However, the stricture origins studied in a 2013 systematic review of current literature were either idiopathic (49\%), iatrogenic (39\%), traumatic (7\%) or inflammatory (6\%) [3].

Iatrogenic causes of stricture include prolonged catheterization or dilatation, traumatic cystoscopy, pelvic radiation, and surgical repair of diverticulum, fistula, or incontinence [4,9]. Iatrogenic cause of stricture can also result from attempts at treating other sources of stricture [7]. This further complicates diagnosis and treatment. Urethral stricture can also result from trauma associated with pelvic fracture or childbirth. In addition, inflammation from recurrent urinary tract infections or carcinoma can cause fibrosis and stricture [4].

In regards to obstructive voiding after anti-incontinence surgery, the etiology can be related to the traumatic nature of the surgery or it can represent a transient sympathetic input $[6,10]$. Suture placement can lead to scarring or maladjustment of the urethra [1]. In addition surgical intervention can lead to increased outlet resistance from "exaggerated urethral compression or hyperelevation of the bladder neck" $[6,7]$.

Midurethral slings (both the traditional retropubic and more recent transobturator approaches) were introduced in 1996 and are now the gold standard in the treatment of female urinary stress incontinence [11,12]. Despite their reported safety and efficacy, complications still exist with both approaches [12]. Retropubic slings may cause irritation of the urethra, with the sling leading to mild obstruction or new onset urgency. Overt obstruction is believed to be due to the elevation of the bladder neck. Postoperative obstruction

\section{Etiologies of Urethral Strictures}

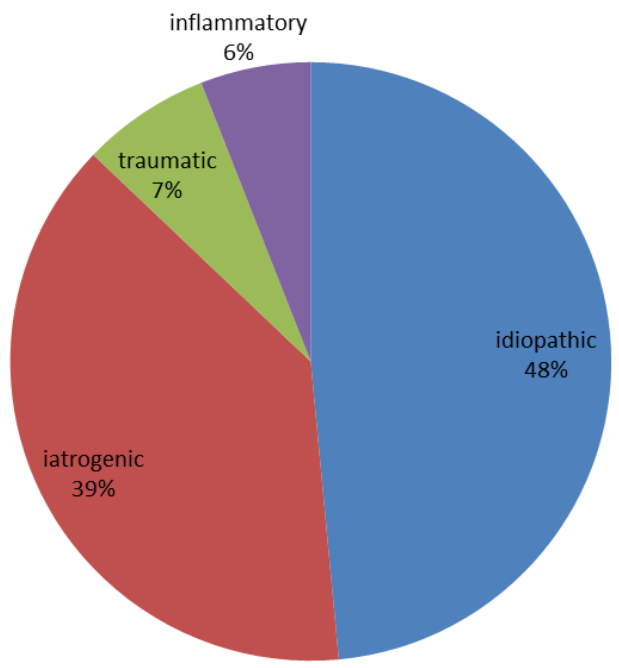

Table 1: Prevalence estimates based upon etiologies reported for 72 patients in a 2013 systematic review of urethral stricture disease (Osman, et al).

Table 2:
\begin{tabular}{|l|}
\multicolumn{1}{|c|}{ Diagnostic Techniques Used for Obstructive Voiding } \\
\hline Urethroscopy* \\
Direct visualization of white scar tissue \\
\hline Voiding cystourethrography* \\
High pressure, low flow rate \\
Radiographic evidence of narrowing \\
\hline Urodynamics \\
Detruser pressure of $25 \mathrm{~cm} \mathrm{H} 2 \mathrm{O}$ \\
Maximum urinary flow rate $<12 \mathrm{~mL} / \mathrm{s}$ \\
\hline AUA symptom scores, Postvoid Residual Volume \\
\hline
\end{tabular}

AUA symptom scores, Postvoid Residual Volume

*Osman, et al, ${ }^{* \star D}$ Defreitas, et al

Table 3:

\begin{tabular}{|l|c|c|}
\hline \multicolumn{3}{|c|}{ Approaches to Treatment of Urethral Stricture Disease } \\
\hline Type of Stricture & Treatment & Success Rate \\
\hline Meatal stricture* & Meatotomy & $96 \%$ \\
\hline \multirow{3}{*}{$\begin{array}{l}\text { Extended Length } \\
\text { Stricture** }\end{array}$} & Dilatation & $47 \%$ \\
\cline { 2 - 3 } & Vaginal flap urethroplasty & $91 \%$ \\
\cline { 2 - 3 } & Vaginal/labial flap & $80 \%$ \\
\cline { 2 - 3 } & $\begin{array}{c}\text { Oral mucosal graft (buccal or } \\
\text { lingual) }\end{array}$ & $94 \%$ \\
\hline
\end{tabular}

*Success rate adopted from study of 50 girls suffering from meatal stenosis (Heising, et al)

**Success rates adopted from systematic review investigating surgical techniques used for 221 patients (Osman, et al)

associated with retropubic slings has been reported ranging from 1.9$19.1 \%$ in previous studies. Transobturator slings have been shown to have lower rates of associated postoperative obstruction. The transobturator approach avoids the retropubic space entirely. This results in decreased rates of complications such as bowel perforation and injury to the bladder and major vessels. Comparatively, these complications are more often seen with placement of retropubic slings [11].

Etiology as well as urethral length and diameter can be very important when determining the proper approach to management of the obstruction caused by stricture or surgical manipulation $[3,4,5,7,13]$.

\section{Diagnosis}

Current studies addressing the diagnosis and treatment of stricture highlight the need for a standard approach to diagnosis. There has been much debate regarding the appropriate diagnostic protocol for post-operative obstruction, as well. Because the symptoms associated with obstruction can suggest broad differential diagnoses, a high index of suspicion is necessary when approaching patients who have experienced urethral manipulation.

Once the possibility of stricture is considered, appropriate tests can help solidify the diagnosis. A recent systematic review revealed that the tests most frequently utilized in the diagnosis of urethral stricture are urethroscopy and voiding cystourethroscopy. Urethroscopy provides direct visualization of stricture, which will appear as a white scar tissue within the urethra. Voiding cystourethrography consistent with stricture would show narrowing of urethra in the area of obstruction [3]. Although these two studies are considered by some authors to be standard diagnostic techniques, there has been recent debate regarding the role of urodynamic studies in developing a diagnosis. This diagnostic tool may prove especially 
Citation: Cockerill K, Canalicio K, DeSouza R. Anatomical Causes of Female Obstructive Voiding: Urethral Stricture and Anti-Incontinence Procedures. J Androl Gynaecol. 2014;2(1): 5.

useful in distinguishing obstruction from stress urinary incontinence and urge incontinence [3]. Osman et al. found that video urodynamic studies consistent with urethral stricture show high pressure, low flow voiding pattern and radiographic evidence of urethral narrowing. In a case-control study involving 82 patients, Defreitas et al. sought to define pressure-flow study cutoff values for anatomic female bladder outlet obstruction. This study reported a detrusor pressure of $25 \mathrm{~cm}$ of $\mathrm{H} 2 \mathrm{O}$ at maximum flow rate and a maximal urinary flow rate of less than $12 \mathrm{ml} / \mathrm{s}$ as consistent with urinary obstruction in females $[4,14]$. However the values obtained in their study still had suboptimal sensitivity and specificity (60-68\%) in identifying bladder outlet obstruction. Because highly specific and sensitive diagnostic tests have yet to be identified, the diagnosis of stricture has often been based on clinical suspicion and direct visualization $[3,9]$.

Osman, et al. suggest that a diagnostic approach with combined analyses could prove more effective. The investigators defined obstruction based on results from urethroscopy and video urodynamics combined with evidence of a urethral calibration of less than 20F. Blaivas et al. suggest calibration with Bougiesa-boule [5]. These diagnostic parameters may provide more descriptive measurement for future studies. Another commonly used measurement for evaluation of obstruction is post void residual [9] Elevated PVR with intact detrusor contractility supports an outflow problem and can be indicative of stricture or obstruction.

Some studies use AUA symptom scores in addition to physical exam measurements to evaluate for stricture. The benefit of this approach lies in the value of comparing symptoms prior to diagnosis to those post-intervention. This allows assessment of the symptomatic effect of treatment. In addition, bladder outlet obstruction was evaluated according to the Abrams-Griffiths nomogram and the Shaeferlin PURR diagram in some studies [15]. These graphical interpretations of outflow obstruction are commonly used for evaluation of male obstruction. The measurements may also assist in evaluating the severity of obstruction in females by comparing maximum flow rate to voiding pressure measured in urodynamic studies. Some studies used these outcomes in determining the patient eligibility for surgery.

Non-invasive uroflowmetry is another useful tool for evaluating obstruction through observing the free flow pattern, or free Qmax. By observing the free flow pattern in relation to detrusor pressure, patient parameters can be graphed and compared to normal nonobstructed values. Particularly, the Liverpool nomogram for women offers reference ranges for comparison of maximum and average urinary flow rate. Additionally, Blaives and Groutz developed a nomogram specific to bladder outlet obstruction in women that can be helpful in grading the severity of obstruction [16].

A high index of suspicion for obstruction should also be placed when patients present with significant lower urinary tract symptoms shortly after anti-incontinence procedures. The standard work-up for post-sling obstruction is not yet established. The role of urodynamics in the diagnosis of obstruction following incontinence surgery has been a recent topic of debate $[17,18]$. Although urodynamics is helpful in establishing the post-surgical diagnosis of obstruction, this method does not contribute to improved outcomes following urethrolysis when compared to diagnosis based on clinical suspicion alone [6]. Women are known to void at low detrusor pressures due to involvement of extraneous muscles. These include abdominal wall flexion and pelvic floor muscle relaxation. This factor may contribute to confounding of diagnosis using urodynamics. Patel and Nitti suggest that radiographic visualization of the urinary tract provides more convincing evidence for diagnosis of obstruction following surgery [1].

Timing of symptoms has been shown to be the best diagnostic indicator for obstruction following anti-incontinence surgery. However, symptoms can occur gradually and sometimes are not evident until more than a year after the procedure date. Mild symptoms can go unreported and progress to more severe complications later in the healing process. For this reason, close evaluation of symptoms and urodynamic evaluation are important steps following antiincontinence surgery to assess for obstruction [12].

Considering the diagnostic techniques used in previous studies, we have found that the best and most definitive approach to urethral stricture and post-surgical obstruction is review of symptoms and direct visualization in a patient with high index of suspicion for outflow tract obstruction. With either diagnosis, it is also important to rule out other causes of urinary symptoms with appropriate tests, such as urinalysis and post void residual volumes [2]. In addition, the urologist should perform a proper physical exam, including genital inspection and bimanual exam to assess for prolapse or a pelvic mass causing obstruction [1]

\section{Management}

There are different approaches to treatment of female urethral stricture. The procedure of choice usually depends on the location of urethral stenosis, the length of proximal healthy urethra, and the integrity of the bladder neck [7]. Depending on these variables, a qualified urologist can treat stricture using urethral dilatation, urethroplasty, vaginal or labial flaps, vaginal or labial grafts, or oral mucosal grafts.

Meatotomy involving longitudinal ventral urethrotomy with transverse closure may be used for confirmed meatal strictures or short strictures. Success rates from a study by Heising, et al. are around $96 \%$ at one year for this treatment [19].

More extensive strictures require a different therapeutic approach. Osman, et al. suggest a primary attempt at dilatation upon diagnosis of stricture. Upon failure of dilatation, the urologist may consider urethroplasty [3]. However, some studies suggest that urethral dilatation is not always the best first line of treatment due to high rates of recurrence as well as worsening of preexisting fibrosis around the urethra [20]. Mean success of urethral dilatation is $47 \%$ [3]. Higher success rates (58\%) are found in those without prior history of dilatation as compared to those with a history (27\%) [3]. Some studies suggest that poor patient compliance with continual intermittent catheterization may contribute to the low success rate for this treatment option $[20,21]$. Complications associated with urethral dilatation procedures include bleeding, extravasation, and further fibrosis. The advantage of this procedure is minimal invasiveness and no significant risk of incontinence [21].

One study suggests that urethroplasty should be performed as first line treatment for stricture disease [20]. In cases utilizing vaginal 
Citation: Cockerill K, Canalicio K, DeSouza R. Anatomical Causes of Female Obstructive Voiding: Urethral Stricture and Anti-Incontinence Procedures. J Androl Gynaecol. 2014;2(1): 5.

flap urethroplasy, success rate is $91 \%$ [3]. Vaginal flap urethroplasty involves the creation of U-shaped or C-shaped flaps of vaginal wall, followed by ventral stricturotomy with suturing of the flap to the sides of the open urethra [22]. A Marthius flap is sometimes recommended when patients require extra support due to inadequate periurethral soft tissue [23]. Vaginal flap urethroplasty involves division of the external urethral meatus. Complications such as inward urinary stream or urinary spraying can result if meatal reconstruction is not properly performed [13]. The advantages of this procedure are its simplicity and the familiar plane of operation for urologists. In addition, there is decreased distortion of vaginal tissues compared to pedicle flap or vestibular flap urethroplasty [22]. However, there is still limited data regarding the use of labial or vestibular flap due to the newness of these procedures.

In cases using vaginal or labial grafts, there is an $80 \%$ success rate. Cases involving oral mucosal grafts have the highest recorded success rate of $94 \%$ [3]. However, buccal mucosal grafting has risk of injury to the parotid duct and mental nerve [13]. For this reason, lingual mucosal grafting offers a lower risk approach [24]. Osman, et al found that local grafting has minimal morbidity. However, buccal or oral mucosal grafting can be very useful when vaginal mucosa cannot be used, as in cases of vaginal fibrosis or atrophy [3].

The technical approach to graft urethroplasty can vary depending on priorities in management. The ventral approach to urethroplasty has the advantage of minimal urethral mobilization during the procedure. However, this approach has increased risk of fistula or injury to the urethra leading to incontinence [3,5]. The dorsal approach to urethroplasty involves dissection of the urethra away from the clitoral cavernous tissue, followed by dorsal stricturotomy and grafting [9]. It has the advantage of strong support as well as ample vascularity supplied by the clitoral tissue. This vascular supply prevents diverticula [25]. This approach can also minimize the risk for sacculations, and it leads to a more physiologic position of the urethral meatus [26]. However, this approach presents with a higher risk of injury to the urethral sphincter and to the neurovascular bundles of the clitoris [27].

Osman et al. suggest that the best approach to urethroplasty is dorsally using a free mucosal graft. However, the small study pools of the articles included in this review present limitations in the strength of analysis. A more recent single-center study exploring dorsal only buccal mucosal graft urethroplasty suggests that this treatment may not be as successful as portrayed in previous small-scale studies. Variations in study pool, study size, and length of time to follow-up could present barriers in comparison of these and other statistics reviewed. In addition, most studies were retrospective in nature and only reflect short term results.

Conservative management of urethral obstruction following anti-incontinence procedures includes foley catheterization or clean intermittent catheterization [10]. Conservative measures are the preferred management within the first week after surgery; however, management following this timeframe diverges [12]. The surgical management of urethral obstruction in this setting includes urethrolysis and simple sling incision [10]. Urethrolysis involves division without direct visualization of the sling. This frees the urethra from the surrounding structures to which it was attached during anti-incontinence surgery. In contrast, simple sling incision involves direct visualization and targeted division of the sling. In the past, treatment was approached with urethrolysis; however recent studies have shown similar effectiveness with simple sling incision. Simple sling incision has also shown fewer risks of significant bleeding, urethral injury, and recurrent SUI as is commonly seen with urethrolysis [28]. A retrospective study looking at women who underwent vaginal midline sling lysis following pubovaginal or midurethral slings showed an advantage in earlier sling lysis (1 year out from surgery). Importantly, there was a low incidence $(8.9 \%)$ of recurrent SUI following this early intervention [29]. Earlier lysis is also beneficial considering the association between delayed treatment of urethral obstruction and irreversible bladder dysfunction [12].

When conservative treatment fails, tansvaginal tape release with or without urethrolysis and segmental tape excision are options as well. With transobturator slings, the associated postoperative obstruction is usually transient and therefore more likely to respond to conservative management alone. In contrast, post-operative obstruction following installment of retropubic sling is more likely to require surgery in order to alleviate symptoms of obstruction. Despite the disparity in outcomes, treatment options are the same for postoperative obstruction following pubovaginal and transobturator slings [11].

\section{References}

1. Patel R, Nitti V (2001) Bladder outlet obstruction in women: prevalence, recognition, and management. Curr Urol Rep 2 : 379-387.

2. Santucci RA, Payne CK, Anger JT, Saigal CS; Urologic Diseases in America Project (2008) Office dilation of the female urethra: a quality of care problem in the field of urology. J Urol 180: 2068-2075.

3. Osman NI, Mangera A, Chapple CR (2013) A systematic review of surgical techniques used in the treatment of female urethral stricture. Euro Urol 64: 965-973.

4. Keegan KA, Nanigian DK, Stone AR (2008) Female urethral stricture disease. Curr Urol Rep 9 : 419-423.

5. Blaivas J, Santos J, Tsui J, Deibert C, Rutman M, et al. (2012) Management of urethral stricture in women. J Urol 188:1778-1782.

6. Aponte MM, Shah SR, Hickling D, Brucker BM, Rosenblum N, et al. (2013) Urodynamic for clinically suspected obstruction after anti-incontinence surgery in women. J Urol 190: 598-602.

7. Tanello M, Frego E, Simeone C, CoscianiCunico S (2002) Use of pedicle flap from the labia minora for the repair of female urethral strictures. Urol Int 69: 95-98.

8. Petri E, Ashok K (2012) Comparison of late complications of retropubic and transobturator slings in stress urinary incontinence. Int Urogynecol J 23: 321 325.

9. Richard Santucci, Mang C (2013) Evaluation and treatment of female urethral stricture disease. Current Bladder Dysfunction Reports 8 :123-127.

10. Campeau L, Al-Afraa T, Corcos J (2008) Evaluation and management of urinary retention after a suburethral sling procedure in women. Curr Urol Rep 9: $412-428$

11. Daneshgari F, Kong W, Swartz M (2008) Complications of mid urethral slings: important outcomes for future clinical trials. J Urol 180:1890-1897.

12. Cetinel B, Tarcan T (2013) Management of Complications After Tension-Free Midurethral Slings. Korean J Urol 54: 651-659.

13. Onol FF, Onol SY, Tahra A, Boylu U (2014) Ventral inlay labia minora graft urethroplasty for the management of female urethral strictures. Urol 83: 460464. 
Citation: Cockerill K, Canalicio K, DeSouza R. Anatomical Causes of Female Obstructive Voiding: Urethral Stricture and Anti-Incontinence Procedures. J Androl Gynaecol. 2014;2(1): 5.

14. Defreitas GA, Zimmern PE, Lemack GE, Shariat SF (2004) Refining diagnosis of anatomic female bladder outlet obstruction: Comparison of pressureflow study parameters in clinically obstructed women with those of normal controls. Urol 64: 675-679.

15. Montorsi F, Salonia A, Centemero A, Guazzoni G, Nava L, et al. (2002) Vestibular flap urethroplasty for stricturesof the female urethra. Impact on symptoms and flow patterns. Urol Int 69:12-16.

16. Blaivas JG, Groutz A (2000) Bladder outlet obstruction nomogram for women with lowerurinary tract symptomatology. Neurourol Urodyn 19: 553-564.

17. Hubeaux K, Deffieux X, Jousse M, Amarenco G (2012) Correlation between voiding dysfunction symptoms and uroflowmetry in women suffering from stress urinaryincontinence. Indian J Urol 28: 313-317.

18. Jeon S, Yoo EH (2012) Predictive value of obstructive voiding symptoms and objectivebladder emptying tests for urinary retention. J Obstet Gynaecol 32: 770-772.

19. Heising J, Seiferth J (1978) Meatus stenosis of girls-clinical demonstration and therapy. Urologe A 17: 292-295.

20. Onol FF, Antar B, Kose O, Erdem M, Onol S (2011) Techniques and results of urethroplasty for female urethral strictures: Our experience with 17 patients. Urol 77: 1318-1324

21. Smith AL, Ferlise VJ, Rovner ES (2006) Female urethral strictures: successfulmanagement with long-term clean intermittent catheterization after urethral dilatation. BJU Int 98: 96-99.
22. Schwender CE, Ng L, McGuire E, Gormley EA (2006) Technique and results of urethroplasty for female stricture disease. J Urol 175: 976-980.

23. Berglund RK, Vasavada S, Angermeier K, Raymond Rackley (2006) Buccal mucosa graft urethroplasty for recurrent stricture of female urethra. Urol 67:1069-1071.

24. Sharma GK, Pandey A, Bansal H, Swain S, Das SK, et al. (2010) Dorsal onlay lingual mucosal graft urethroplasty for urethral strictures in women. BJU Int 105:1309-1312.

25. Singh M, Kapoor R, Kapoor D, Kapoor R, Srivastav A, et al. (2013) Dorsal onlayvaginal graft urethroplasty for female urethral stricture. Indian $\mathrm{J}$ Urol 29:124-128.

26. Migliari R, Leone P, Berdondini E, De Angelis M, Barbagli G, et al (2006) Dorsal buccal mucosagraft urethroplasty for female urethral strictures. J Urol 176:1473-1476.

27. Goel A, Paul S, Dalela D, Sankhwar P, Sankhwar SN, et al. (2014) Dorsal onlaybuccal mucosal graft urethroplasty in female urethral stricture disease: a single-center experience. Int Urogynecol J 25: 525-530.

28. South MM, Wu JM, Webster GD, Weidner AC, Roelands JJ, et al. (2009) Early vs late midline sling lysis results in greater improvement in lower urinary tract symptoms. Am J Obstet Gynecol 200: 564.

29. Moore CK, Goldman HB (2013) Simple sling incision for the treatment of iatrogenic bladder outlet obstruction. Int Urogynecol J 24: 2145-2146. 